\section{Arousal Reaction in EEG induced by Blood Pressure}

Ir is supposed by several authors ${ }^{1-9}$ that adrenalineinduced EEG-arousal, which originates in the mesencophalic reticular formation and the posterior hypothalamus, is caused by a direct action of adrenaline on these structures. Discharges were recorded from single cells within the posterior hypothalamus ${ }^{10,11}$. The firing-rate of these cells was affected by intravenous injection of adrenaline or by mechanically induced changes of blood pressure. There is no effect of adrenaline if the blood pressure is kept constant artificially. We therefore tested the hypothesis that the adrenaline-induced arousal is a secondary effect of the rising blood pressure.

The following experiments were performed on cats with an encéphale isolé preparation. It was observed that the EEG-arousal after intravenous injection of adrenaline occurred about I sec after the beginning of the rise in blood pressure. Fig. 1 shows that an arousal is also obtained after an increase of blood pressure, which is induced mechanically by a short, strong injection of Ringer solution into the aorta. The high, slow-voltage pattern simultaneously in all leads changes to a fast low activity, which is typical for an arousal reaction. In this case, the arousal occurs at the end of the injection artefact, when the blood pressure has started to rise. Rothballer' found a cortical arousal on injection of adrenaline into the mesencephalic reticular formation without changes in the systemic blood pressure. This seoms to support the hypothesis of a local action of adrenaline. In our experiments, a local injection of mammalian Ringer solution into the same area also caused a cortical arousal. Therefore, Rothballer's observation might be explained by a stimulation of the cells induced by the mechanical stimulus of the injection.

It can be concluded from our observations that an increase of blood pressure can excite the ascending reticular activating system. The 'pressosensitive area', described by Baust et al." is possibly responsible for these effects. This area can be excited by changes in systemic blood pressure as well as by local pressure stimuli as shown in the experiment mentioned

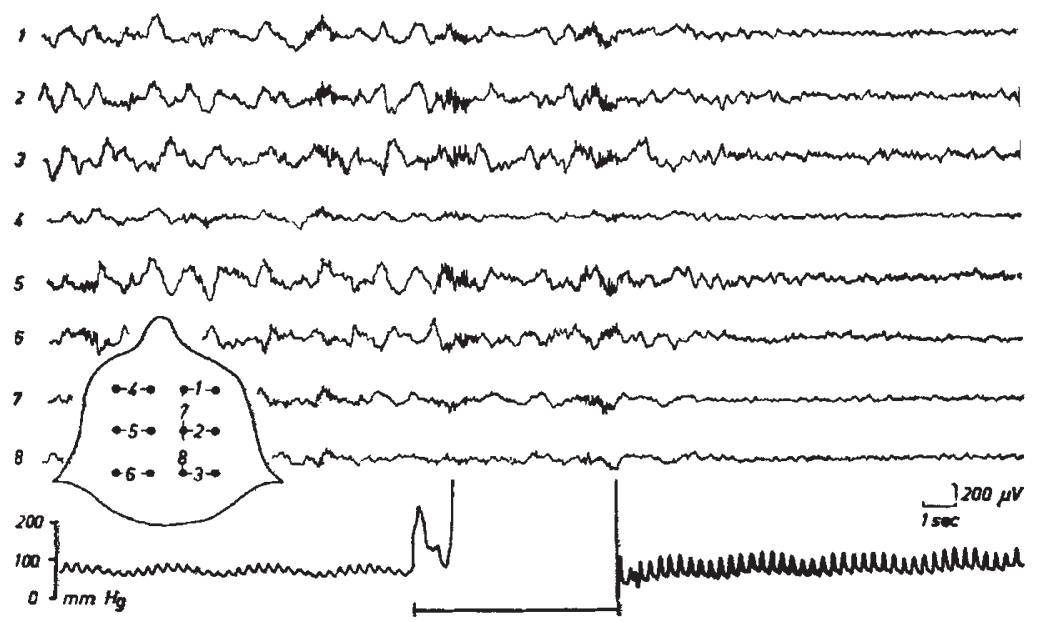

Fig. 1. Encephale isole preparation of the cat, no narcotics present. Artificial respiration. Lines 1-8, bipolar EEG-records from the cortex; location of electrodes as indicated in the inset. Lowest line, simultaneous blood pressure record in the femoral artery. During
the mark (below), $10 \mathrm{ml}$. of mammalian Ringer solution were injected into the aorta here. The fact that injection of vasopressin leads to EEG-arousal ${ }^{4}$ also favours our hypothesis.

\section{W. BaUsT \\ H. NIEMCZYK \\ J. VIETH}

Physiologisches Institut,

Universität, Heidelberg.

Bonvallet, M., Hugelin, A., and Dell, P., J. Physiol. (Paris), 48, $403(1956)$

Bonvallet, M., Dell, P., and Hugelin, A., J. Physiol. (Paris), 46,

.

Capon, A., J. Physiol. (Paris), 50, 201 (1958).

Dell, P., Adrenergic Mechanisms, CIBA Found. Symp., 393 (London, $1960)$.

- Jasper, H., Biological and Biochemical Bases of Behaviour, 37 (Madi-

' Krupp, P., Pfilgers Arch., 273, 499 (1961).

Mantegazzini, P., Poeck, K., and Santibanez-H, G., Arch. Ital. Biol., (1959).

Rothballer, A. B., EEG, 8, 603 (1956)

${ }^{10}$ Baust, W., and Katz, P., Pfügers Arch., 272, 575 (1961).

Baust, W., Niemczyk, H., Schaefer, H., and Vieth, J., Pfiugers

\section{Serum Cholesterol in Rats intoxicated with Carbon Tetrachloride}

ORAL administration of carbon tetrachloride to rats causes an elevation in the amount of $\beta$-globulins while reducing the albumin, $\alpha_{1^{-}}$and $\alpha_{2}$-globulins. Subsequent injury by subcutaneous sponge implantation of the intoxicated rats augments both $\alpha_{2}$ - and $\beta$ regions ${ }^{1}$. Since the $\alpha_{2^{-}}$and $\beta$-globulin regions of rat serum contain lipoproteins ${ }^{2}$, the fatty infiltration of the liver might be a responsible factor in some of the changes in electrophoretic patterns which are observed in injured, intoxicated rats. Carbon tetrachloride poisoning is apparently associated with a reduced rate of lipoprotein synthesis resulting in a lowering of the plasma lipoprotein-level and an accumulation of liver lipids ${ }^{3}$. Preliminary experiments, however, showed no intensification of lipoprotein staining (sudan black) of the paper electropherograms obtained with serum from injured, carbon tetrachlor. ide intoxicated rats.

To test this point further, male white rats (Holtzman) were treated by oral administration of $0.1 \mathrm{ml}$. of carbon tetrachloride mixed with mineral oil (1.1 mixture) per $100 \mathrm{~g}$ of body-weight for a total of six days. Some of the poisoned rats were afterwards injured by the subcutanoous implantation (dorsal) of a 200-mg piece of 'Ivalon' sponge after the fifth dose of carbon tetra. chloride; a sixth dose was given $24 \mathrm{~h}$ later. Normal control rats, control rats receiving $0.1 \mathrm{ml}$. of mineral oil in six daily doses and rats injured by sponge implanta. tion only accompanied the experiments with carbon tetrachloride.

$24 \mathrm{~h}$ after the sixth dose of carbon tetrachloride or mineral oil or $48 \mathrm{~h}$ after sponge implantation, the rats were anæsthetized with ether and bled by cardiac puncture. The livers were per. fused with physiological saline solu. tion, excised and homogenized with an equal volume of dis. tilled water. The homogenates 